\title{
Electrical characteristics of amorphous molybdenum-nickel contacts to silicon
}

\author{
K. T-Y. Kung, I. Suni, ${ }^{\text {a) }}$ and M-A. Nicolet \\ California Institute of Technology, Pasadena, California 91125
}

(Received 15 July 1983; accepted for publication 30 January 1984)

\begin{abstract}
The electrical characteristics of sputtered, amorphous Mo-Ni contacts have been measured on both $p$ - and $n$-type $\mathrm{Si}$, as functions of composition (30, 54, and 58 at. \% Mo). The contact resistivity on both $p^{+}$and $n^{+} \mathrm{Si}$ is in the $10^{-6} \Omega \mathrm{cm}^{2}$ range. The barrier height for as-deposited samples varies between $\phi_{b p}=0.47-0.42 \mathrm{~V}$ on $p$-type Si and between $\phi_{b n}=0.63-0.68 \mathrm{~V}$ on $n$-type $\mathrm{Si}$, as the composition of the amorphous layer goes from Ni-rich to Mo-rich. The sum $\phi_{b p}+\phi_{b n}$ always equals $1.12 \mathrm{~V}$, within experimental error. After thermal treatment at $500^{\circ} \mathrm{C}$ for $\frac{1}{2} \mathrm{~h}$, the contact resistivity changes by a factor of two or less, while the barrier height changes by at most $\sim 0.05 \mathrm{~V}$. In light of these results, the amorphous Mo-Ni film makes good ohmic contacts to silicon.
\end{abstract}

PACS numbers: 73.40.Cg, 73.40.Ns, 73.30. $+\mathrm{y}$

Finding thermally stable metallizations for making contacts to semiconductors is of critical importance in device technology. At elevated temperatures, the electrical characteristics of the contacts can be undesirably altered by the formation of an intermixed layer, due to atomic interdiffusion across the metal/semiconductor interface. To limit contact degradations of this type, diffusion barriers offer attractive options. ${ }^{1}$ For diffusion barrier applications, amorphous metallic layers may perform better than polycrystalline films, ${ }^{2-4}$ primarily because of the absence of grain boundaries and dislocations, which dominate the low-temperature diffusion process in polycrystalline films. ${ }^{5}$ A good amorphous diffusion barrier requires structural and chemical stability, as well as low contact resistivity to act as an effective electrical contact to the semiconductor substrate.

So far, the only amorphous alloy whose electrical contact properties have been reported is sputtered $\mathrm{Fe}_{45} \mathrm{~W}_{55}{ }^{6}$ : its contact resistivity is in the $10^{-6}-\left(10^{-7}\right) \Omega \mathrm{cm}^{2}$ range on $p^{+}$ $\left(n^{+}\right)$Si, and remains constant after thermal treatment up to at least $500^{\circ} \mathrm{C}, \frac{1}{2} \mathrm{~h}$. The Mo-Ni system has been chosen for this study because: (i) Mo and Ni follow the structural difference rule for amorphous phase formation ${ }^{7}$; sputtered $\mathrm{Mo}-\mathrm{Ni}$ films are in fact amorphous over a wide range of composition: from 20 to 70 at. $\% \mathrm{Mo}^{8}$; (ii) these amorphous films are thermally stable: no polycrystalline phase can be observed in samples deposited on $\mathrm{Si}$, after $1 \mathrm{~h}$ annealing at $525^{\circ} \mathrm{C}$ and below ${ }^{3}$; and (iii) $\mathrm{Mo}, \mathrm{Ni}$, and their silicides have been used as contacts, or as IC interconnects, on $\mathrm{Si}$, and their electrical characteristics are readily available ${ }^{9}$ for comparison to the amorphous Mo-Ni contacts. In this communication the contact resistivity of amorphous Mo-Ni films, $\rho_{c}$, on $p^{+}$, and $n^{+}$ $\mathrm{Si}$, and the Schottky barrier height, $\phi_{b}$, on $p$ - and $n$-type Si are reported as functions of composition.

The contact resistivity measurements were performed at room temperature on a test pattern reported previously [Fig. 1(b) of Refs. 6, 10, and 11]. Starting with $1 \Omega \mathrm{cm},\langle 100\rangle$ Si wafers, $\mathrm{a} \mathrm{SiO}_{2}$ layer of $\sim 4000-\AA$ thickness was thermally grown, diffusion windows were opened by standard photo-

\footnotetext{
Permanent address: Semiconductor Laboratory, Technical Research Centre of Finland, Otakaari 5A, SF-02150, Espoo 15, Finland.
}

lithography, the heavily doped $\left(N_{A} \cong 10^{20} \mathrm{~B} / \mathrm{cm}^{3}\right.$; $\left.N_{D} \cong 2 \times 10^{20} \mathrm{P} / \mathrm{cm}^{3}\right)$ shallow $\left(x_{j} \cong 0.3 \mu \mathrm{m}\right) p^{+}$and $n^{+}$layers $\left(R_{s} \cong 30-40 \Omega / \square\right)$ were formed, and the metal contacts were then patterned by lift-off. For the barrier height measurements, circular diodes of two differing diameters $(d$ mask $=0.5$ and $1.0 \mathrm{~mm})$ were fabricated on $1-3 \Omega \mathrm{cm} p$ type and $2-4 \Omega \mathrm{cm} n$-type $\langle 100\rangle \mathrm{Si}$ wafers, also by lift-off. Mo-Ni films for both $\rho_{c}$ and $\phi_{b}$ measurements were obtained simultaneously by rf cosputtering from a composite target. Prior to the deposition, the samples were dipped into a dilute ( $10 \%) \mathrm{HF}$ solution, dried in $\mathrm{N}_{2}$-gas flow, and loaded immediately into the sputtering system. The sputtering was performed under $\sim 15 \mathrm{~m}$ Torr Ar pressure, at $\sim 400 \mathrm{~W}$ and $\sim 350-500 \mathrm{~V}$ target voltage. The Mo-Ni deposition continued at a rate of $\sim 30-35 \AA / \mathrm{min}$ for $\sim 30-45 \mathrm{~min}$. Three sets

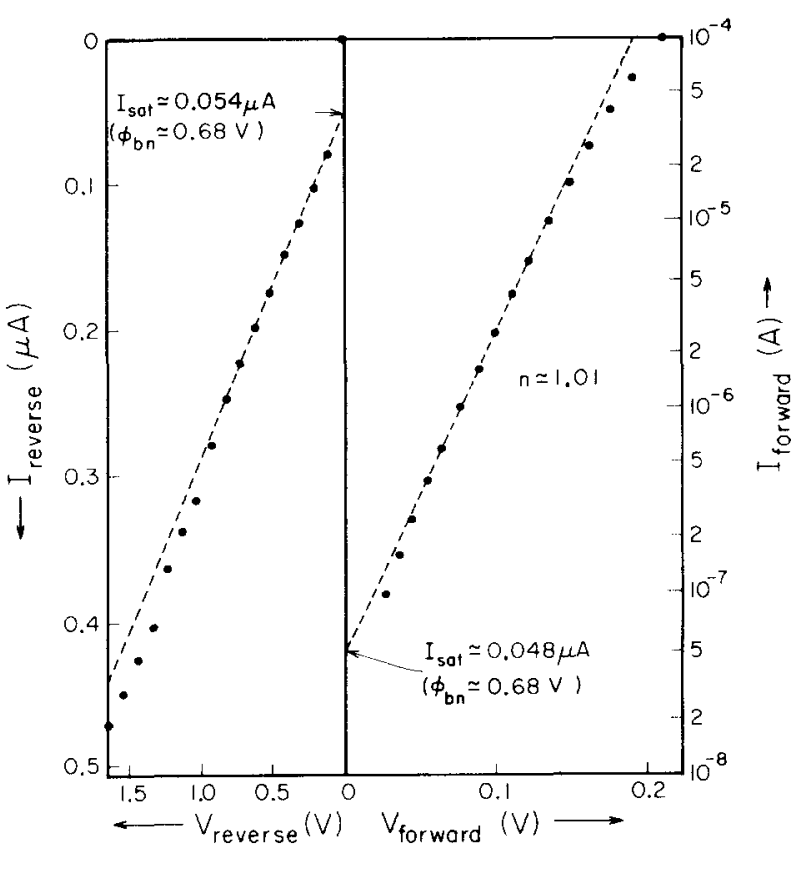

(a)

(b)

FIG. 1. Reverse (a) and forward (b) $I-V$ characteristics measured at room temperature on a diode with a $0.0019 \mathrm{~cm}^{2}$ area of amorphous $\mathrm{Mo}_{58} \mathrm{Ni}_{42}$ on $2-4 \Omega \mathrm{cm},\langle 100\rangle, n$-Si substrate in the as-deposited state. 
of samples of differing Mo-Ni compositions were made following the same procedures. Compositions of the three layers were determined by backscattering spectrometry (BS); their amorphous structure was established by $\mathrm{x}$-ray diffraction (Read camera).

The barrier height of the amorphous Mo-Ni contact on Si was determined from conventional $I-V$ measurements. ${ }^{9}$ Figure 1(b) shows the forward characteristics obtained for amorphous $\mathrm{Mo}_{58} \mathrm{Ni}_{42}$ as deposited on $n$-type Si. Note that the $I-V$ curve deviates from the exponential approximation (dashed line), due to reverse leakage current, at $V<0.05 \mathrm{~V}$, and due to the bulk series resistance, at $V>0.15 \mathrm{~V}$. However, a straight line can be drawn through the $\log I$ vs $V$ data at intermediate points that show high linearity. The current intercept, extrapolated to $V=0$, was taken as the forward saturation current.

Figure 1(a) shows the reverse characteristics measured on the same diode. Extrapolation of the reverse curve to $V=0$ yields approximately the same saturation current that was already derived from the forward characteristics. The reverse current, however, does not saturate at high biases, but instead shows a linear dependence on voltage. We suspect that this is due to additional leakage mechanisms which act collectively as a shunt resistance in parallel with the diode. The back contact which was made using an alloy of In and $\mathrm{Ga}$ may also have added to the nonideal reverse behavior.

The barrier height $\phi_{b}$ was calculated from the saturation current, $I_{\text {sat }}=$ (diode area) $A^{* *} T^{2} \exp -q \phi_{b} / k T$, assuming Richardson's constant $A_{e}^{* *} \cong 112 \mathrm{~A} / \mathrm{cm}^{2} / \mathrm{K}^{2}$ for electrons and $A_{h}^{* *} \cong 32 \mathrm{~A} / \mathrm{cm}^{2} /{ }^{\circ} \mathrm{K}^{2}$ for holes. ${ }^{9}$

For samples on $n$-type substrates, both forward and reverse measurements were performed and, since the results are reasonably consistent, only the average value is reported as the barrier height in Table I, along with the average ideality factor from the forward measurement. As a check, the barrier height was also evaluated from the forward characteristics using the method established by Norde. ${ }^{12}$ The results are in good agreement with the values given in the table. Since the $p$-type barrier height is low, the effect of substrate series resistance is enhanced by the resulting high current in the forward direction. Therefore, the $p$-type barrier height was determined from the reverse measurement alone. The resulting $\phi_{b p}$ is also shown in Table I. In addition, the barrier heights of $\mathrm{Mo}, \mathrm{Ni}$, and their silicides quoted in the literature are given in the same table for comparison.

The measured barrier heights of as-deposited, amorphous Mo-Ni samples can be considered reasonable since the relationship $\phi_{b p}+\phi_{b n}=E_{\text {gap }, \mathrm{Si}}=1.12 \mathrm{~V}$ is satisfied, within error of measurement. The ideality factor values indicate that good diode behavior has been observed. To determine their thermal stability, the barrier heights were determined on the same sets of samples after vacuum annealing at $500^{\circ} \mathrm{C}$ for $1 / 2 \mathrm{~h}$. The remeasured values are also shown in Table I. In general, a change is observed for each composition either for the $p$ - or the $n$-type barrier, and the sum $\phi_{b p}+\phi_{b n}$ decreases by $0.03-0.05 \mathrm{~V}$. The lowering of the effective barrier heights is attributed to (1) a laterally nonuniform metal-semiconductor interface after annealing, ${ }^{13}$ and (2) an increase in the effective contact area due to an irregular interface morphology.

Table I shows that after annealing the barrier heights converge toward common values $(0.4$ and $0.6 \mathrm{~V})$ regardless of the layer composition. This result suggests the formation of a common interfacial layer. Backscattering spectrometry spectra indeed show an interfacial reaction in all samples.

TABLE I. Measured barrier heights and ideality factors of sputtered Mo-Ni Schottky diodes. The barrier heights for Ni, Mo, and their silicides on $n$-type Si are found in the literature and are given here as reference.

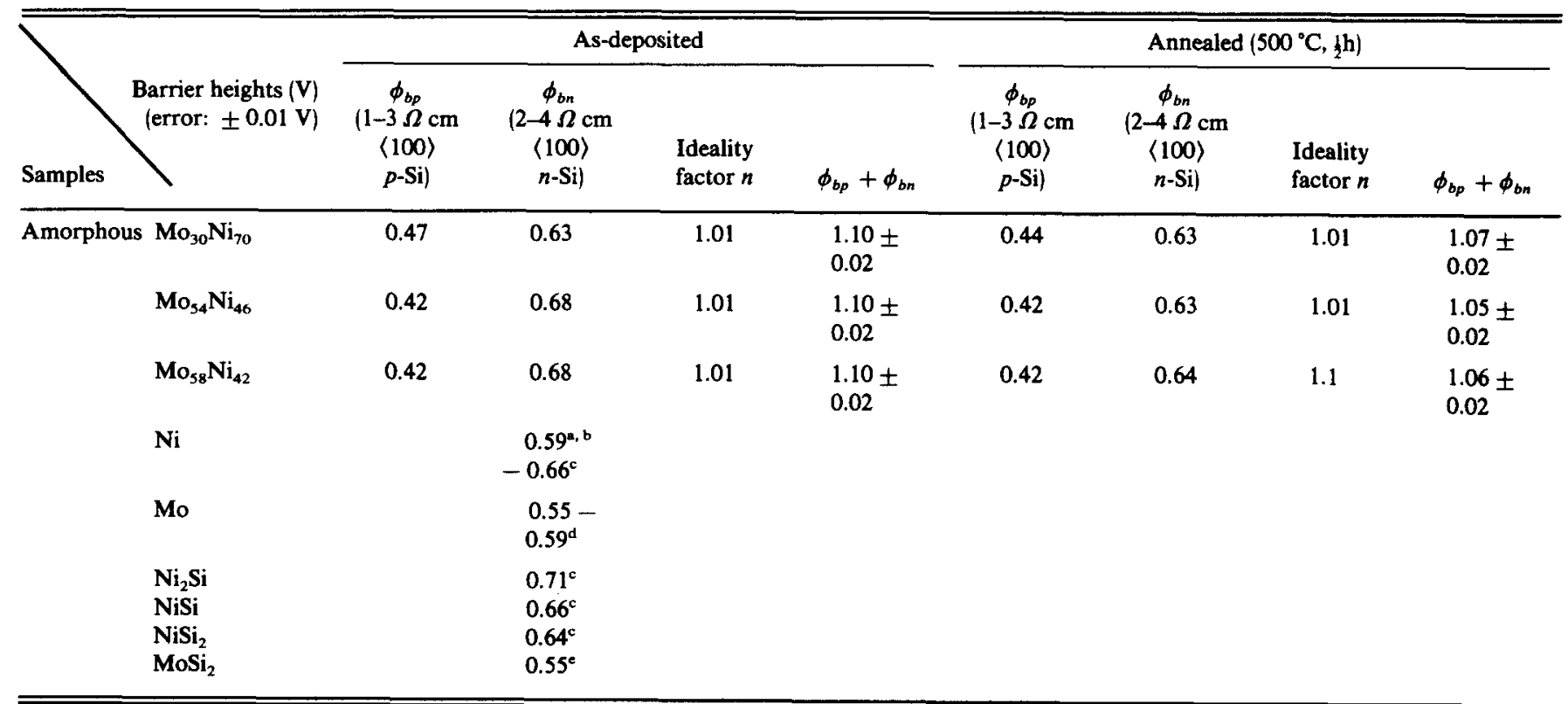

J. M. Shannon, Appl. Phys. Lett. 24, 369 (1974).

'M. Finetti, I. Suni, and M-A. Nicolet, J. Electron. Mater. (to be published).

'T. R. Harrison, A. M. Johnson, P. K. Tien, and A. H. Dayem, Appl. Phys. Lett. 41, 734 (1982).

${ }^{d}$ E. H. Rhoderick, Metal-Semiconductor Contacts (Clarendon, Oxford, 1980), p. 53.

e J. M. Andrews and J. C. Phillips, Phys. Rev. Lett. 35, 56 (1975). 
TABLE II. Calculated and measured contact resistivities of sputtered amorphous Mo-Ni films on Si. Values for Ni and Mo reported in the literature are given as reference.

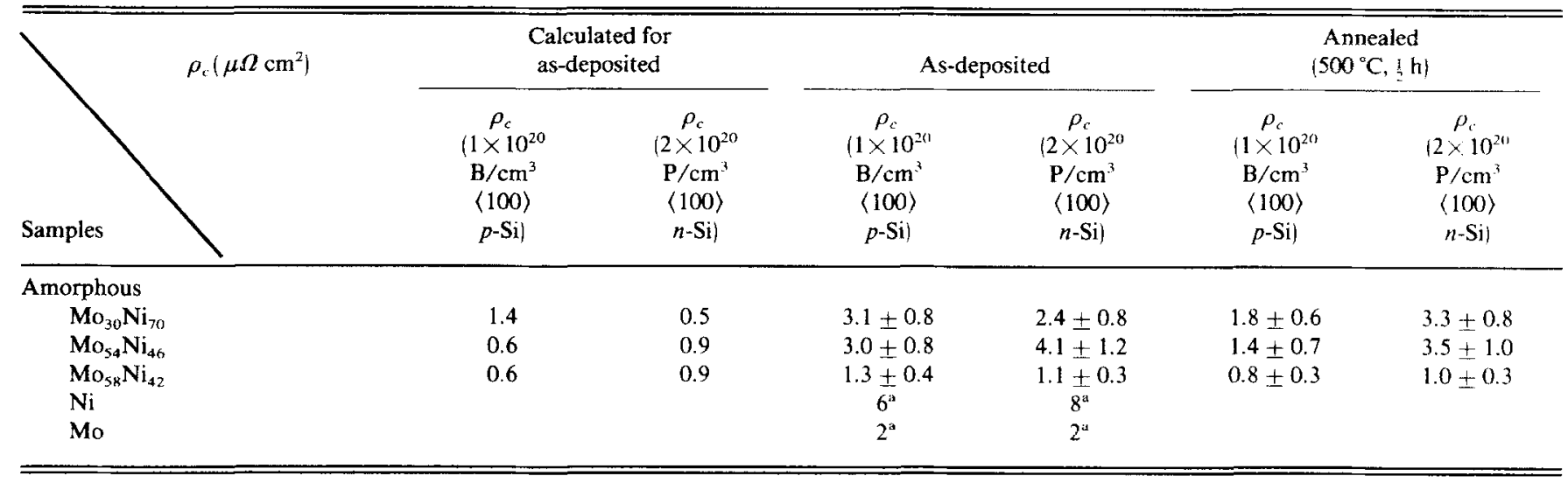

${ }^{2}$ Lewis E. Terry and Richard W. Wilson, Proc. IEEE 57, $1580(1969) .\left(N_{A} \cong 0.7 \times 10^{20} \mathrm{~B} / \mathrm{cm}^{3} ; N_{D} \cong 0.6 \times 10^{20} \mathrm{P} / \mathrm{cm}^{3}.\right)$

This reaction is most pronounced for the Ni-rich films; there, an interfacial layer can be identified that consists essentially of $\mathrm{Ni}$ and $\mathrm{Si}$ only. The evolution of this reaction at $600{ }^{\circ} \mathrm{C}$ suggests that this layer is $\mathrm{NiSi}$, whose barrier height agrees with the value measured after annealing. Preferential reactions of this sort have previously been reported for crystalline compound films. ${ }^{14-17}$ In contrast to those cases, the Mo-Ni films considered here are still amorphous after annealing at $500^{\circ} \mathrm{C}$. This and the fact that Mo is a high-temperature silicide former, explain the high temperatures needed to induce a reaction. Further studies are necessary to firmly establish the nature and outcome of this reaction.

We have also measured the contact resistivities of our amorphous films using the linear transmission line model (LTLM) approach. ${ }^{18}$ The procedures for the measurement and the evaluation of the contact resistivity established in Ref. 19 have been followed here. This evaluation technique takes into account the effect of the finite sheet resistance of the metallic contact layer. In our case, the contact resistivity value derived directly from the conventional LTLM calculation, without considering the metal sheet resistance, would deviate from the true value by at least one order of magnitude. The contact resistivity so obtained is presented in Table II as a function of composition. A theoretical contact resistivity can be calculated ${ }^{20}$ for each sample, from the measured barrier height and the surface dopant concentration of the heavily doped layer, assuming an effective mass $m_{e}^{*} \cong 0.5$ $m_{o}$ for electrons or $m_{h}^{*} \cong 0.66 m_{o}$ for holes. ${ }^{21}$ The contact resistivities of amorphous Mo-Ni so calculated, along with those of sputtered Mo and Ni measured previously, are shown together in Table II. It can be seen that the values measured in this work are in good agreement with the theoretical predictions. Furthermore, the contact resistivities of amorphous $\mathrm{Mo}-\mathrm{Ni}$ on $\mathrm{Si}$ are very close to those of pure Mo and pure $\mathrm{Ni}$, if the difference in sample dopings are taken into account. This is an expected outcome since the barrier height of amorphous Mo-Ni on Si differs little from that of either Mo or Ni. With tunneling being the predominant mode of current transport, a small difference in barrier height will not result in a significant change of the contact resistivity.
The contact resistivities were also remeasured from the same sets of samples after thermal treatment at $500^{\circ} \mathrm{C}$ for $\frac{1}{2}$ $h$, and the results are given in Table II. They are seen to remain in the same order of magnitude as those of the asdeposited samples. This is again expected since the barrier heights do not change much (at most $\sim 0.05 \mathrm{~V}$ ) upon annealing.

In summary, the electrical characteristics of amorphous Mo-Ni contacts on silicon have been studied by measuring the Schottky barrier heights and the contact resistivities on both $n$-type and $p$-type substrates. Only small changes are observed in the barrier heights and contact resistivities after vacuum annealing at $500{ }^{\circ} \mathrm{C}$ for $\frac{1}{2} \mathrm{~h}$. The changes are attributed to the formation of a common interfacial layer that consists essentially of $\mathrm{Ni}$ and $\mathrm{Si}$ only. We conclude that amorphous binary metal alloys of the type represented by the Mo-Ni case are attractive alternatives for primary ohmic contacts to shallow $\mathrm{Si}$ junction devices.

The authors are grateful to $M$. Finetti for assistance in using the photolithographic facilities and for constructive criticisms on the electrical measurements, B. X. Liu and T. Banwell for assistance in operating the $\mathrm{X}$-ray diffractometer and the $\mathrm{MeV}$ van de Graaff accelerator, and R. Gorris for technical assistance. Access to the photolithography lab and to the x-ray diffraction facility were granted by Professor D. Rutledge and Professor T. Vreeland, Jr. This work was supported in part by the U.S. Department of Energy through an agreement with the National Aeronautics and Space Administration and monitored by the Jet Propulsion Laboratory, California Institute of Technology (D. B. Bickler and D. R. Burger).

'M-A. Nicolet, Thin Solid Films 52, 415 (1978).

${ }^{2}$ M-A. Nicolet and M. Bartur, J. Vac. Sci. Technol. 19, 786 (1981).

${ }^{3}$ J. D. Wiley, J. H. Perepezko, J. E. Nordman, and Kang-Jin Guo, IEEE Trans. Ind. Electron. IE-29, 154 (1982).

${ }^{4}$ B. L. Doyle, P. S. Peercy, R. E. Thomas, J. H. Perepezko, and J. D. Wiley, in Interfaces and Contacts, edited by $R$. Ludeke and K. Rose, MRS Symposia Proceedings, Vol. 18 (North-Holland, New York, 1983), p. 69.

${ }^{5}$ D. Gupta, D. R. Campbell, and P. S. Ho, in Thin Films-Interdiffusion 
and Reactions, edited by J. M. Poate, K. N. Tu, and J. W. Mayer (Wiley, New York, 1978), p. 161.

${ }^{6}$ M. Finetti, E. T-S. Pan, I. Suni, and M-A. Nicolet, Appl. Phys. Lett. 42, $987(1983)$.

${ }^{7}$ B. X. Liu, W. L. Johnson, M-A. Nicolet, and S. S. Lau, Appl. Phys. Lett. 42, $45(1983)$.

${ }^{8}$ R. P. W. Lawson, W. A. Grant, and P. J. Grundy, Nucl. Instrum. Methods 209/210, 243 (1983).

"See, for example, S. M. Sze, Physics of Semiconductor Devices, 2nd ed. (Wiley, New York, 1981), Chap. 5.

${ }^{10} \mathrm{M}$. Finetti, I. Suni, and M-A. Nicolet, Solar Cells 9, 179 (1983).

"M. Finetti, I. Suni, and M-A. Nicolet, Solid-State Electron. 26, 1065 (1983).

${ }^{12}$ H. Norde, J. Appl. Phys. 50, 5052 (1979).
${ }^{13}$ I. Ohdomari and K. N. Tu, J. Appl. Phys. 51, 3735 (1980).

${ }^{14}$ K. N. Tu, W. N. Hammer, and J. O. Olowolafe, J. Appl. Phys. 51, 1663 (1980).

${ }^{15}$ M. Eizenberg, G. Ottaviani, and K. N. Tu, Appl. Phys. Lett. 37, 87 (1980).

${ }^{16}$ K. N. Tu, J. Vac. Sci. Technol. 19, 766 (1981).

${ }^{17}$ G. Ottaviani, K. N. Tu, W. K. Chu, L. S. Hung, and J. W. Mayer, J. Appl. Phys. 53, 4903 (1982).

${ }^{18}$ H. H. Berger, Solid-State Electron. 15, 145 (1972).

${ }^{19}$ M. Finetti, P. Ostoja, S. Solmi, and G. Soncini, Solid-State Electron. 23, 255 (1980).

${ }^{20}$ A. Y. C. Yu, Solid-State Electron. 13, 239 (1970).

${ }^{21} \mathrm{C}$. Y. Chang, Y. K. Fang, and S. M. Sze, Solid-State Electron. 14, 541 (1971). 